\title{
A Generic Approach for Team Selection in Multi- player Games using Genetic Algorithm
}

\author{
S. M. Aqil Burney \\ Department of Computer \\ Science, University of \\ Karachi, Karachi-75270,
}

Pakistan

\author{
Nadeem Mahmood \\ Department of Computer \\ Science, University of \\ Karachi, Karachi-75270, \\ Pakistan
}

\author{
Kashif Rizwan \\ Department of Computer \\ Science \& IT, Federal \\ Urdu University, Karachi, \\ Pakistan
}

\author{
Usman Amjad \\ Department of Computer \\ Science, University of \\ Karachi, Karachi-75270, \\ Pakistan
}

\begin{abstract}
The best team selection is always the requirement of management in different domains and in different organizations including government, project, industry, business and sports. The traditional team selection process is really lengthy, awkward and unclear due to manual process and personal judgments; which may lead to a disaster. These conflicting constraints and personal judgments can be translated into software for better and quick solution.
\end{abstract}

This paper presents a solution to this problem with the help of genetic algorithm to find the optimal solution for the problem of cricket team selection and formation. Our approach is the combination of the existing quantitative approaches with some new extensions such as attributes regarding personal performances, team performance and the combination of players. Secondly our method is just not specific for cricket team but it is converted to generic model for other multiplayer games. We propose an adaptation of island genetic algorithm to optimize the selection of multiplayer sports team having multiple conflicting constraints with mixed crossover where the fitness of common solution is used to drive the selection.

\section{General Terms}

Genetic Algorithm, Sports, Optimization.

\section{Keywords}

Player Selection, Genetic Computing, Multi-Player Sports, Team Selection, Cricket.

\section{INTRODUCTION}

Process of team selection in multi-player sports is a complex decision due to many factors. The performance of the team depends upon the combination of team players selected with respect to the performance, event, opponent and the playing conditions. The confidence of the team is dependent on various factors which may include the recent performance of the players, the quality and fairness of the game, satisfaction of selection board or committee including coaches and captain; moreover the spectators. Aforesaid factors significantly improve the chances of win with an optimal team selection. Team Selection in multiplayer sports such as football, hockey and cricket is a daunting task. Composition of the team depends on many factors which include the player's individual performance, combination of players, physical fitness, playing conditions and above all, the confidence of the selection committee on the players. Selection of players in a team is always a difficult decision making task and it has many dimensions. There are finite set of variables with many constraints. The best team selection is a real world problem for sports management and associations especially in multi-player sports. Team selection problem may rise in various domains i.e., software project team, in HRM for promotion, software testing criterion and bench mark selection etc.

The manual team selection process may have many drawbacks such as personal liking and disliking, personal grudges between team selection committee and players and external pressures (social, political etc.). Poor team selection lead to failure and the selection committee and sports organizing authorities will become accountable to the people who love sports. It also effects the loyalty of players due to non-optimal and substandard selection of combination; causing global damages for the side. The team selection procedure also takes and ample amount of time and chances of defacement exists. This may results in a bad performance in the game and the morale of the whole team shattered. To avoid this vague practice of team selection a scientific and more effective procedure can give an optimistic result in a lesser amount of time [1]. We have proposed a software based solution for the optimal team combination by using evolutionary programming technique called genetic algorithm [2].

We have applied our proposed GA for the selection of cricket team as it is one of the most popular games in South-East Asia and specifically in India and Pakistan. Cricket team is comprised of 11 players categorized as bowlers, batsman and wicket keeper. However there are other categories as well such as all-rounder (a bowler who can bat or vice versa). Similarly batsman can be categorized as (top order or middle order). Cricket has different formats test cricket (5 day match), one day international (50 over match) and T-20 (20 over match). Team selections are based on the format of the cricket a player may be rated well for test cricket may not be considered for one day international or vice versa. Very few research papers found in the literature regarding GA in team selection, however [4] and [7] presented GA based solution for optimal cricket team selection.

The next section gives a brief introduction of genetic algorithm and the problem definition. Next section presents the flow chart of applying genetic algorithm to team selection process. Finally the players data is processed using the propose model and results are displayed.

\section{GENETIC ALGORITHM IN A NUT SHELL}

Evolutionary algorithms such as genetic algorithm may be used for optimization of real world problems. Genetic Algorithms (GAs) [2], [3], [8] and [9] are adaptive heuristic search algorithm designed to simulate processes in natural system necessary for evolution where we have a huge search space. GAs compared to the traditional methods, provides an 
alternative methods to solving problem by outperforming the classical methods in many problems domain [6].

Team selection is also an optimization problem as the selection committee looks for the best combination available with given constraints. GA, as discussed earlier considered being the best tool for optimization in such problems [5]. GA with its power of computation can solve this problem in a much desirable and efficient manner which can make the job of selection committee much easier.

Let's consider a pool of 100 players and the task of the selection committee to select 15 players for the competition. There may be many combinations of best team players, provided the realities and constraints. Selecting the best team (optimal) combination is the main objective and we also call this the fittest combination. The procedure of finding the fittest combination is a time consuming task and the resultant or the survivor among the fit candidates leads. In other words, we have to find the "Survivor of the fittest".

\subsection{Working of GA in Context}

GA works by initializing the population (Chromosomes) as elaborated below (see Figure 1). Secondly, Selection is made randomly (Rolette wheel selection algorithm may be used to avoid the influence of initial population), using the fitness function. Now the process of Crossover is to be made and at last Mutation (Optional). This will be repeated in a cycle till the user intervene or fittest combination found.

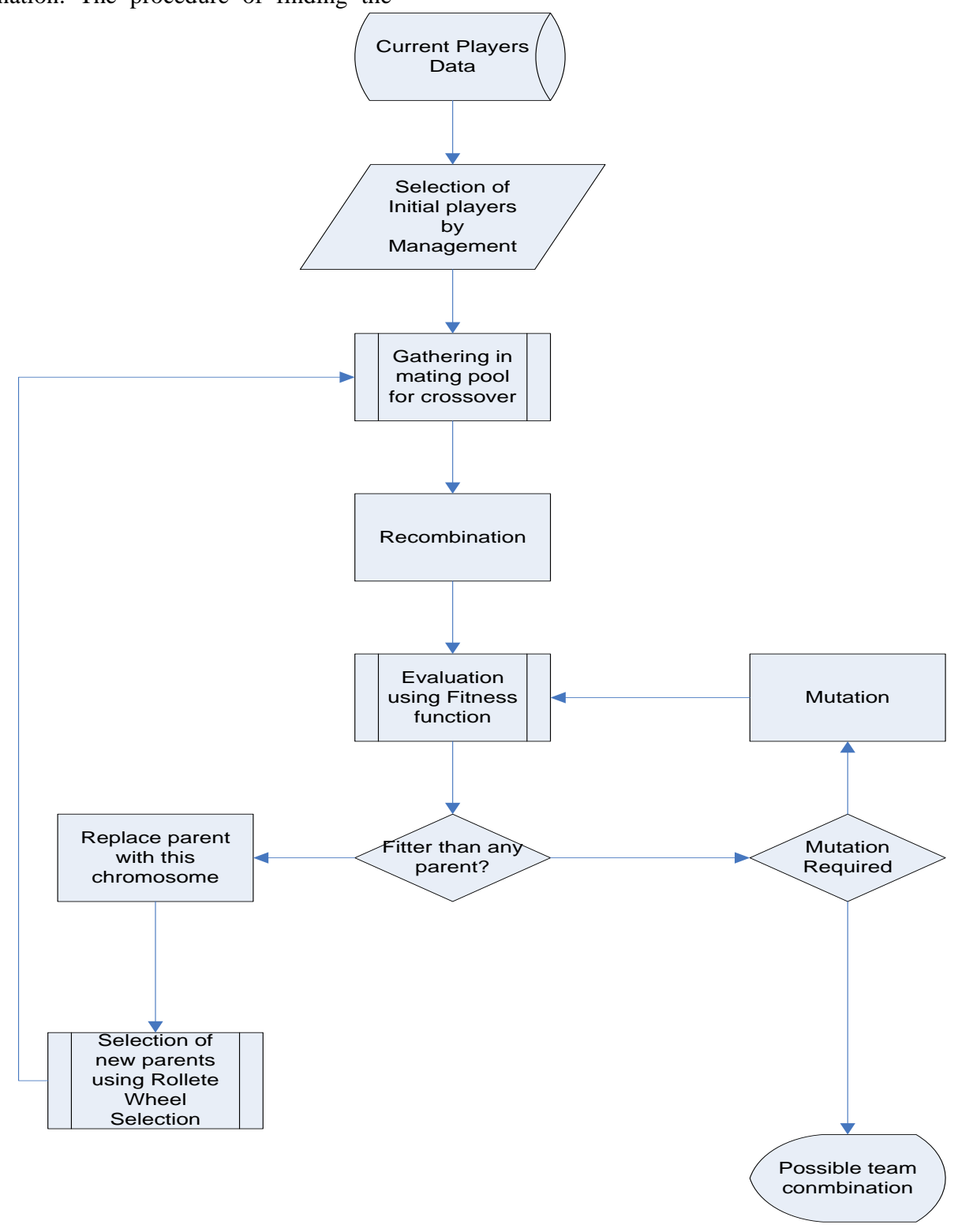

Fig 1: Procedural Flow for proposed method of team selection using GA

Nevertheless, inspired by natural phenomenon, heuristics, and techniques, the Genetic Algorithms (GAs) are, so far, the better in finding the solution of problems in the domain where; very little knowledge base is available. GA is a unfussy yet strong algorithm which can work very well in a variety of environments. Therefore very little priory knowledge is enough to find a coherent solution. The solution provided by GA is in fact brings a good quality. Here, feasibility evaluation is required to find the best solution for any specific problem provided by the GA analogy i.e., selection, crossover and/or mutation. 


\subsection{Limitation and Way Out of GA}

Besides many strong problem solving strengths, GAs are superseded by any other modal specific algorithms, where universe of discourse is not complex, so, this is in fact very true that it has boundaries as well and eventually not successful in every situation [6]. One other issue is their time complexity which makes it not suitable for the real time applications. But to override this problem, we may pose the implementation of this Modality in parallel computation; to reduce the time complexity limitations and attain success.

GA launched with all candidate solutions. A Model of modal is required to be formulated so the fitness or the degree of happiness can be calculated for feasible solution. Feasible solutions in the universe of discourse are collected based on following major steps.

a. Evaluation or the degree of fitness is tagged up to respective candidate after calculation. Initially, various pairs of candidate solutions are selected with higher degree of fitness. These candidate solutions are then dispatched to the mating pool for Crossover.

b. Mutation is performed if necessary.

Step-'a' \& step-'b' continue-on in iteration till a suitable, necessary and sufficient solution with a particular number of generations have attained. That is again based on the degree of happiness for the specific situation. Evaluation and cost of fitness function may be matter of concern and settlement standard is the modality when better candidates are not improving well; after various iteration.

$$
\operatorname{Pr}\left(k_{i}\right)=\frac{\operatorname{Fitness}\left(k_{i}\right)}{\sum_{j=1}^{n} \operatorname{Fitness}\left(k_{j}\right)}
$$

Where,

$$
\operatorname{Pr}\left(k_{i}\right)_{=} \text {fitness of current } \mathrm{k}^{\text {th }} \text { chromosome }
$$

$\mathrm{n}=$ number of genes or size of chromosome (i.e., total number of players)

$\mathrm{i}=$ number of currently available players

$\mathrm{j}=$ Number of all available players

An approach to designate a fair chance to the best candidates is mentioned in equation (i). Better generations are replaced with if parents are not better.
GA is allowed to evolve until best chromosome is not obtained. Stopping criteria of GA is discussed in next section.

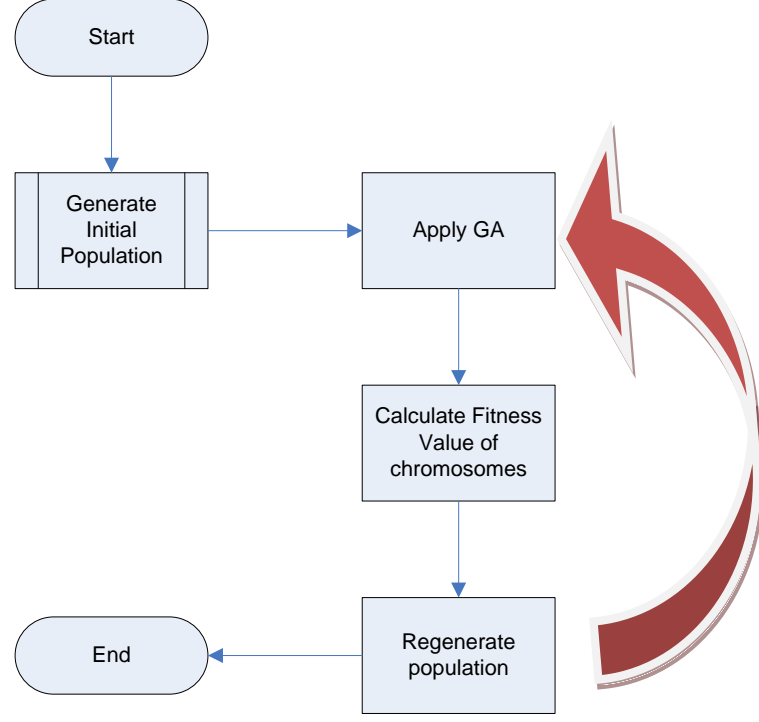

Fig 2: Modular approach for team selection using GA

\subsection{Generate Initial Population}

In the proposed method we have made initial population of 10 chromosomes, initialized randomly. Our chromosomes contains randomly generated player IDs from the available set of players.

\subsection{Applying GA}

After generation of initial population, Crossover and Mutation operations are applied. Crossover is applied on two randomly selected chromosomes from our population. Similarly Mutation is applied on a randomly selected chromosome with a mutation rate of 0.5

\subsection{Calculate Fitness Values of Chromosomes}

In the next step fitness values of chromosomes are calculated using equation (ii) and equation (iii).

\subsection{Regenerate Population}

New population is generated using best 5 chromosomes from population based on fitness values calculated in above step. Other five chromosomes are newly generated randomly to complete set of 10 chromosomes.

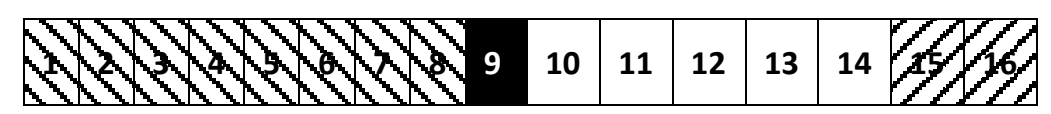

Fig 3: Structure of team using GA approach

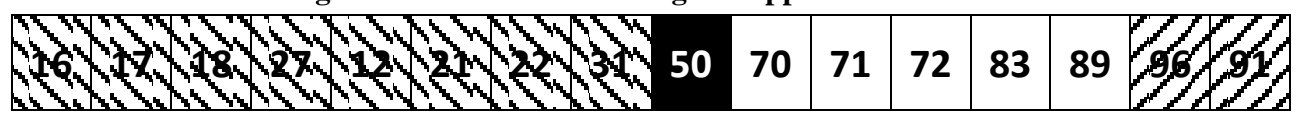

Fig 4: Calculated one of the best chromosome for proposed team of 16 players

\section{MODULAR APPROACH FOR TEAM \\ SELECTION USING GA}

Eventually, a modular approach as shown in (see Figure 2) is proposed to adopt appropriate optimization technique considering different possibilities in aforesaid team selection problem [8]. 


\section{GA FOR PLAYER SELECTION}

Team is a combination of different types of players (see Figure 3) which shows a sample chromosome for a team of 16 players with 4 types of player categories. The description of squad is further elaborated in Table-1.

$$
\begin{aligned}
& \mathrm{W}=\text { number of type-1 players. (Gene } 1 \text { to Gene } 8) \\
& \text { ( } W=8 \text { in our case) } \\
& \mathrm{X}=\text { number of type-2 players. (Gene } 9 \text { ) } \\
& (X=1 \text { in our case }) \\
& \mathrm{Y}=\text { number of type-3 players. (Gene } 10 \text { to Gene } 14 \text { ) } \\
& (Y=5 \text { in our case } \\
& \mathrm{Z}=\text { number of type- } 4 \text { players. (Gene } 15 \text { to Gene } 16 \text { ) } \\
& (\mathrm{Z}=\mathbf{2} \text { in our case) }
\end{aligned}
$$

Table 1: Description of cricket team squad (16 players)

\begin{tabular}{|c|l|c|c|}
\hline S. No. & Type of player & Description & No. Of players \\
\hline 1. & Type-1 & Specialized batsman & 8 \\
\hline 2. & Type-2 & Wicket keeper & 1 \\
\hline 4. & Type-3 & Specialized bowler & 5 \\
\hline 3. & Type-4 & All rounder & 2 \\
\hline
\end{tabular}

\subsection{Fitness Function}

$$
\begin{aligned}
F P_{i} & =\frac{1-\frac{(G L)_{i}}{(G P)_{i}}+(L M W)_{i}}{1+(L M W)_{i}} \\
\bar{F}_{k} & =\sum_{i}^{n} F P_{i} / n
\end{aligned}
$$

Where,

$\overline{F_{k}}=$ Average fitness of $\mathrm{k}^{\text {th }}$ chromosome

$\mathbf{n}=$ Number of genes or size of chromosome (i.e., total number of players) (16 in above case)

$\mathbf{i}=$ Number of available players

$(\mathbf{F P})_{\mathrm{i}}=$ Fitness of $\mathrm{i}^{\text {th }}$ player

$(\text { GP })_{i}=$ Number of games played by $i^{\text {th }}$ player (gene)

$(\mathbf{G L})_{\mathbf{i}}=$ Numbers of games lose by ith player (gene)

$(\mathbf{L M W})_{\mathrm{i}}=$ Number of games won by $\mathrm{i}^{\text {th }}$ player (gene)

\subsection{Resolving Tie between two players}

If a tie occurs at some point during experiment, i.e., two or more players with same fitness value gather in the mating pool, it will be resolved using fitness values and experience values of players in tie or a Heuristic as given below:

$$
\Delta \text { Experience }=E\left(P_{i}\right)-E\left(P_{j}\right)
$$

$$
\partial=\frac{|\Delta f(P)|}{\Delta \text { Experience }}
$$

Where,

$$
\begin{aligned}
& \mathbf{E}(\mathbf{P})_{i}=\text { experience(fitness) of } i^{\text {th }} \text { player } \\
& \mathbf{E}(\mathbf{P})_{j}=\text { experience(fitness) of } j^{\text {th }} \text { player } \\
& \Delta \mathbf{f}(\mathbf{P})=\text { difference in fitness value of current pair } \\
& \text { of player (having tie) }
\end{aligned}
$$

\begin{tabular}{|c|c|}
\hline Player ID & Fitness Value (FP) \\
\hline 1 & 0.532307692 \\
\hline 2 & 0.786259542 \\
\hline 3 & 0.896296296 \\
\hline 4 & 0.948717949 \\
\hline 5 & 0.931712963 \\
\hline 6 & 0.905555556 \\
\hline 7 & 0.587443946 \\
\hline 8 & 0.918604651 \\
\hline 9 & 0.602409639 \\
\hline 10 & 0.466666667 \\
\hline 11 & 0.892335766 \\
\hline 12 & 0.902702703 \\
\hline 13 & 0.863636364 \\
\hline 14 & 0.916666667 \\
\hline 15 & 0.785714286 \\
\hline 16 & 0.769230769 \\
\hline 17 & 0.860759494 \\
\hline 18 & 0.9296875 \\
\hline 19 & 0.631578947 \\
\hline 20 & 0.919831224 \\
\hline 21 & 0.927350427 \\
\hline 22 & 0.916666667 \\
\hline 23 & 0.777777778 \\
\hline 24 & 0.727272727 \\
\hline 25 & 1 \\
\hline 26 & 0.75 \\
\hline
\end{tabular}

\section{Results \& Discussion}

There may be various combinations exist; pitching an optimal solution is inevitable. Therefore, genetic algorithm like heuristic algorithm may be tailored to solve this problem. We have applied proposed method on currently available players of Pakistan cricket team, shown above in Table 3. Based on our proposed fitness function of GA, we obtained fitness values of all players shown below in Table 2 . Based on these fitness values following team combination is obtained shown below in Table 4 and (see Figure 4) that shows best chromosome evolved after 2257 generations of GA. Our obtained fitness value for this chromosome is 0.9544220740625 .

Table 2: Fitness values of players (calculated w.r.t. table-2) 
Table 3: History of Pakistan Cricket Players for fitness evaluation

\begin{tabular}{|c|c|c|c|c|c|c|}
\hline $\begin{array}{l}\text { Player } \\
\text { ID }\end{array}$ & Player Name & $\begin{array}{l}\text { Matches } \\
\text { Played }\end{array}$ & Won & Lost & $\begin{array}{l}\text { No. of } \\
\text { wins } \\
\text { (last M } \\
\text { matches) }\end{array}$ & Specification \\
\hline 1 & Shahid Afridi & 325 & 173 & 152 & 0 & ALL \\
\hline 2 & Abdul Razzaq & 262 & 143 & 112 & 1 & ALL \\
\hline 3 & Fawad Alam & 27 & 13 & 14 & 4 & BAT \\
\hline 4 & Misbah-ul-Haq & 78 & 52 & 24 & 5 & BAT \\
\hline 5 & Mohammad Yousuf & 288 & 164 & 118 & 5 & BAT \\
\hline 6 & Umar Gul & 90 & 55 & 34 & 3 & BOW \\
\hline 7 & Younis khan & 223 & 125 & 92 & 0 & BAT \\
\hline 8 & Umar Akmal & 43 & 21 & 21 & 5 & BAT \\
\hline 9 & M. Sami & 83 & 48 & 33 & 0 & BOW \\
\hline 10 & Saeed Ajmal & 45 & 21 & 24 & 0 & BOW \\
\hline 11 & Kamran Akmal & 137 & 74 & 59 & 3 & WC \\
\hline 12 & Imran Farhat & 37 & 19 & 18 & 4 & BAT \\
\hline 13 & Taufeeq Umar & 22 & 13 & 9 & 2 & BAT \\
\hline 14 & Sohail Tanvir & 36 & 23 & 12 & 3 & BOW \\
\hline 15 & Asad Shafiq & 21 & 12 & 9 & 1 & BAT \\
\hline 16 & Azhar Mahmood & 143 & 73 & 66 & 1 & ALL \\
\hline 17 & Imran Nazir & 79 & 45 & 33 & 2 & BAT \\
\hline 18 & Shoaib Malik & 192 & 106 & 81 & 5 & ALL \\
\hline 19 & Ahmed Shehzad & 19 & 11 & 7 & 0 & BAT \\
\hline 20 & Mohammad Hafeez & 79 & 39 & 38 & 5 & BAT \\
\hline 21 & Salman Butt & 78 & 43 & 34 & 5 & BAT \\
\hline 22 & Mohammad Asif & 38 & 16 & 19 & 5 & BOW \\
\hline 23 & Mohammad Amir & 15 & 5 & 10 & 2 & BOW \\
\hline 24 & Wahab Riaz & 22 & 15 & 6 & 0 & BOW \\
\hline 25 & Sarfaraz Ahmed & 6 & 3 & 0 & 4 & WC \\
\hline 26 & Zulqurnan Haider & 2 & 2 & 0 & 1 & WC \\
\hline
\end{tabular}

Table 4: Proposed team combination as per GA approach

\begin{tabular}{|l|}
\hline Sarfaraz Ahmed \\
\hline Misbah-ul-Haq \\
\hline Mohammad Yousuf \\
\hline Shoaib Malik \\
\hline Salman Butt \\
\hline Mohammad Hafeez \\
\hline Umar Akmal \\
\hline Sohail Tanvir \\
\hline Mohammad Asif \\
\hline Umar Gul \\
\hline Imran Farhat \\
\hline Fawad Alam \\
\hline Kamran Akmal \\
\hline Taufeeq Umar \\
\hline Imran Nazir \\
\hline Abdul Razzaq \\
\hline
\end{tabular}


We fixed stopping criteria for GA that if no change in fitness value occurs after 10000 iterations then stop. (see Figure 5) which shows convergence graph of GA. (see Figure 6) that shows variations in GA fitness values with respect to iterations. Best chromosome is obtained after 2257 generations.

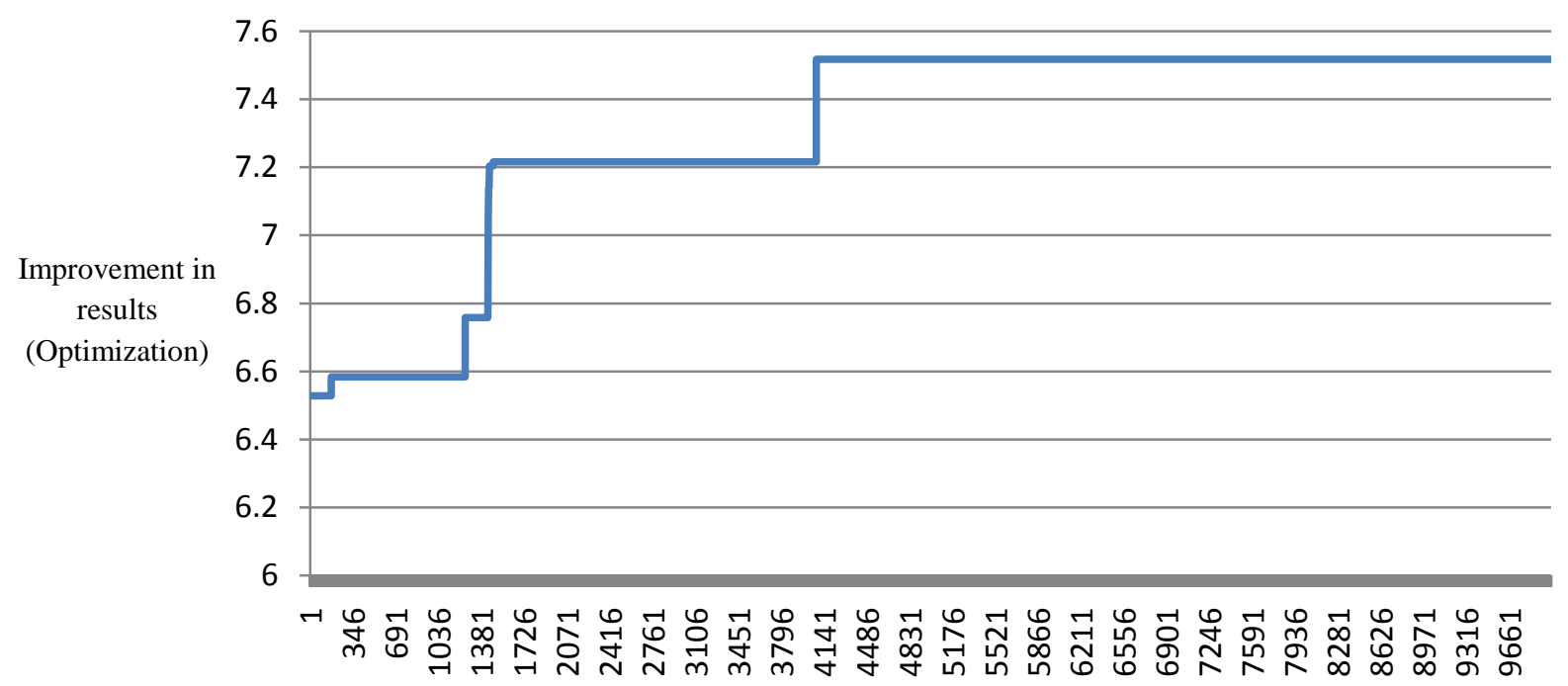

No. of Iterations of GA

Fig 5: An evaluation of GA computation on players' data (using table-2 \& table-3)

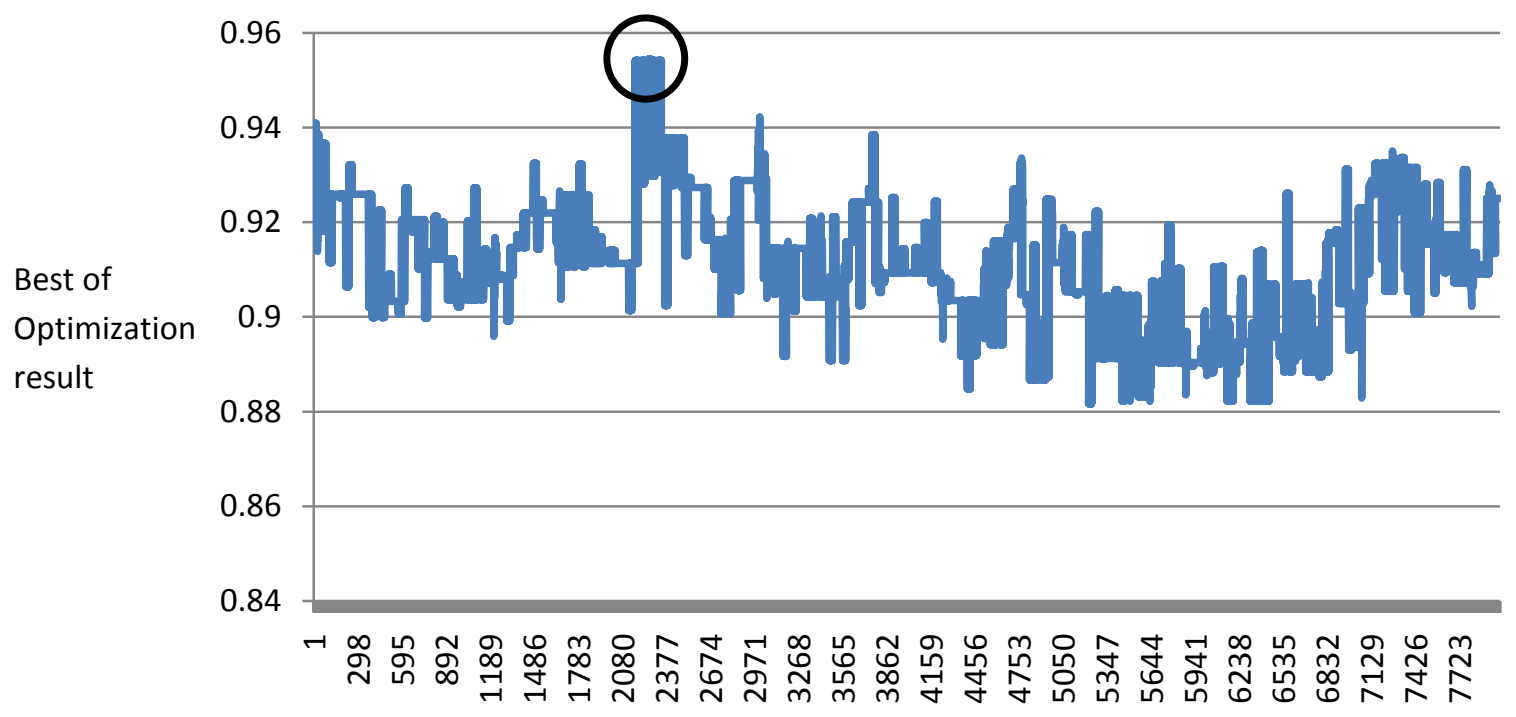

No. of Iterations of GA

Fig 6: A map of variations in computation of GA fitness values 


\section{CONCLUSION AND FUTURE WORK}

A generic approach for multi player team selection has been developed using genetic algorithm. This automated process will select team on the basis of the previous outcomes i.e. either win or lose. This algorithm also takes care of the recent performance of the players by considering last $\mathrm{M}$ matches. We have applied this algorithm to cricket team selection; however it can be applied to any multiplayer sports. There are constraints which are specific to sports and this algorithm is flexible to accommodate those constraints. We have tested the algorithm on bunch (top) of Pakistani cricket players for test team selection. The results indicate the usefulness of the proposed algorithm. Extended version of above mentioned work with complex and specific domain with a flavor of constraint satisfaction problem solving technique would be more effective. Furthermore the individual performance of the player may also be included for team selection process.

\section{REFERENCES}

[1] B. Feng, Z. Jiang, Z. Fan, N. Fu, "A method for member selection of cross-functional teams using the individual and collaborative performances". European Journal of Operational Research. June 16, 2010;203(3):652-661. Available from: Business Source Complete, Ipswich, MA.

[2] J. R. Koza, Genetic Programming. Cambridge, MA: (1992) The MIT Press/Bradford Books.
[3] Melanie Mitchell. An introduction to genetic algorithms. MIT Press, Cambridge, Massachusetts, 1996.

[4] S. N. Omkar, "Cricket team selection using genetic algorithm", in: Proceedings of the International Congress on Sport Dynamics, Melbourne, Australia, (2003), pp. 1-9.

[5] A. Popov, Genetic Algorithms for optimization User Manual, 2005, Hamburg.

[6] F. Rothlauf. Representations for Genetic and Evolutionary Algorithms. Springer, Heidelberg, $2^{\text {nd }}$ edition 2006.

[7] S. Siva Sathya Md. Shad Jamal, "Applying Genetic Algorithm to Select an Optimal Cricket Team". International Conference on Advances in Computing, Communication and Control (ICAC3'09), January 2324, 2009, Mumbai, Maharashtra, India.

[8] Khan Z. A., Burney S. M. Aqil, Naseem J., Rizwan K., "Optimization of Power Consumption in VLSI Circuit", IJCSI International Journal of Computer Science Issues, Vol. 8, Issue 2, March 2011.

[9] G. Guttman, M. Shpitalni, "Linear Programming and Genetic Algorithms Methods for Creation of Groups in Networks of Excellence", CIRP Annals - Manufacturing Technology, Volume 55, Issue 1, 2006, Pages 143-146. 